\title{
Investigating solvability and complexity of linear active networks by means of matroids
}

\section{Petersen, Bjørn}

Published in:

IEEE Transactions on Circuits and Systems

Link to article, DOI:

10.1109/TCS.1979.1084645

Publication date:

1979

Document Version

Publisher's PDF, also known as Version of record

Link back to DTU Orbit

Citation (APA):

Petersen, B. (1979). Investigating solvability and complexity of linear active networks by means of matroids. IEEE Transactions on Circuits and Systems, 26(5), 330-342. https://doi.org/10.1109/TCS.1979.1084645

\section{General rights}

Copyright and moral rights for the publications made accessible in the public portal are retained by the authors and/or other copyright owners and it is a condition of accessing publications that users recognise and abide by the legal requirements associated with these rights.

- Users may download and print one copy of any publication from the public portal for the purpose of private study or research.

- You may not further distribute the material or use it for any profit-making activity or commercial gain

- You may freely distribute the URL identifying the publication in the public portal 


\title{
Investigating Solvability and Complexity of Linear Active Networks by Means of Matroids
}

\author{
BJORN PETERSEN
}

\begin{abstract}
The solvability and complexity problems of linear active networks are approached from a purely combinatorial point of view, using the concepts of matroid theory. Since the method is purely combinatorial, we take into account the network copology alone. Under this assumption necessary and sufficient conditions are given for the unique solvability of linear active networks. The complexity and the number of dc-eigenfrequencles are also given.

The method enables you to decide if degeneracies are due to the topology alone, or if they are caused by special relations among network parameter values. If the network parameter values are taken into account, the complexity and number of de-eigenfrequencies given by the method, are only upper and lower bounds, respectively.

The above conditions are fairly easily checked, and the complexity and number of dc-eigenfrequencies are found, using polymomially bounded algorithms (matroid partition and intersection algorithms).
\end{abstract}

\section{INTRODUCTION}

$I^{1}$ N THE THEORY of $R L C$-networks without controlled sources, combinatorial methods have been known for many years, which solve the following two essential problems.

1) Decide if a certain network is solvable (i.e., if the network equations have a unique solution).

2) In case of a solvable network find a set of independent state variables.

The graph theoretical tool is known as the normal tree method. This expression was originally used by Kuh and Rohrer [9] although the method is identical to that of Bryant [3]. The basic observation of this combinatorial method is, that the topology alone (i.e., the network graph and the type and position of the network elements) is sufficient to answer 1) and 2). These answers are not affected by any specific choice of the network parameter values.

If $R L C$-networks contain controlled sources then, in general, the topology and a specific choice of network parameter values must be taken into consideration answering 1) and 2). In most approaches this involves calculating the determinants of large matrices, although methods have been given to reduce the size of these matrices [4]. To determine the complexity, the normal tree method has been used to give a preliminary set of state variables [5], and conditions have been given for the increase or decrease of the complexity [6]. If a network is not solvable (in the following the network is then said to

Manuscript received July 22, 1977, April 21, 1978.

The author is with the Department of Mathematics, Technical University of Denmark, DK 2800 Lyngby, Denmark.

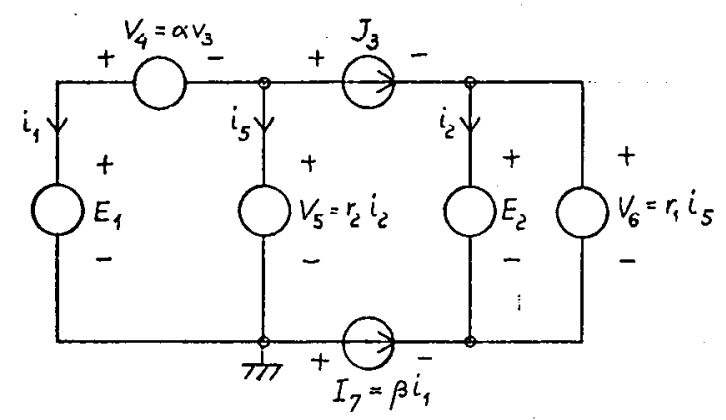

Fig. 1. The network $N_{1}$ of Example (1.1) and Example (6.3).

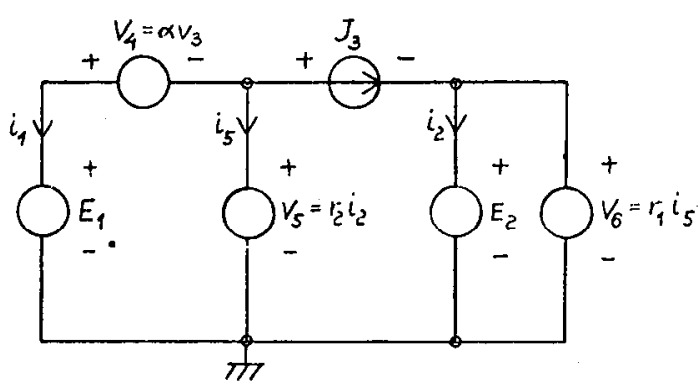

Fig. 2. The network $N_{2}$ of Example (1.2) and Example (6.4).

be singular) it is not possible however, by the above methods, to decide if the singularity is due to the topology or a specific choice of network parameter values.

In the following, examples are given, illustrating these topics.

\section{Example (1.1)}

The network $N_{1}$ of Fig. 1 is singular due to the topology alone (this is verified in Section VI).

In most cases, however, singularity is also due to a specific choice of network parameter values. This is illustrated by the following example.

\section{Example (1.2)}

The network $N_{2}$ of Fig. 2 is solvable except for $\alpha=-1$ and/or $r_{1}=0$ and/or $r_{2}=0$ (this is verified in Section VI).

In the same way the number of independent state variables, i.e., the complexity, may be restricted exclusively by the topology or by a specific choice of network parameter values. 
It has been the subject of many papers to develop methods making it possible to determine if singularity is due to the topology alone, and to give a better upper bound on the complexity, than the number of capacitors and inductors. In [11] a generalized tree is introduced to obtain sufficient conditions for capacitor voltages and inductor currents to be state variables. In [7], [14], and [21] the concept of two graphs is considered. The concept of two graphs was originally introduced by Mayeda [12], but [7], [14], and [21] cover a wider class of networks. In [7], [14], and [21] the network element descriptions are given, if not explicitly then essentially, by a hybrid immittance matrix. To each nonzero element in the hybrid immittance matrix a controlled and a controlling edge is introduced, which may increase the number of edges considerably, and two graphs, different from the network graph, is defined. In [7] and [21] necessary and sufficient conditions are given for solvability and complexity of linear active networks. Furthermore, [7] gives necessary and sufficient conditions for the existence of hybrid immittance descriptions. In [7] the conditions are given a matroid interpretation, which enables a unifying algorithmic approach, using the algorithm which finds a base in the union of matroids. In the present paper we will go one step further and use matroids, not only in the interpretation of the conditions, but in the mathematical model itself.

The constellation of electrical networks and matroids has been considered to be a very useful combinatorial tool in electrical network theory for some years [13]. The author was introduced to the subject through a seminar directed by Recski, Research Institute for Telecommunication, Budapest, during his visit to Denmark in the autumn of 1975. The basic idea of Recski [18] was the introduction of the union of matroids as a tool in the theory of linear active networks. The models of [19], however, treated current and voltage constrains separately, thus neglecting the algebraic constrains forced upon currents and voltages by, e.g., resistors. These models, therefore, did not allow the formulation of necessary and sufficient conditions for solvability and complexity.

The extension of the method of Recski [19], which is the subject of this paper, was conceived as a part of the author's M.Sc. thesis [15] during the spring of 1976. The basic idea of this model, is the introduction of two copies of the edge set of the network graph. The main new features is that the model introduce no additional elements, treat network element descriptions given by any algebraic matrix (not necessarily a hybrid immittance matrix; thus the model treat, e.g., norators and nullators), maintain a close relation between the network graph and the matroids treating the graphic constrains and introduce (by means of the union of matroids) the concept of a network matroid which give compact conditions, easily checked by polynomially bounded algorithms. Apart from necessary and sufficient conditions for solvability, complexity, and existence of hybrid immittance descriptions, we give necessary and sufficient conditions for the existence of any prescribed description. Furthermore, a modification proposed by the author [16], of the algorithm of [8], give the zero and nonzero entries of the corresponding matrices.

The paper contains more material than necessary for the explanation of the basic ideas in the mathematical model. It is the hope of the author, however, that this relatively detailed presentation will be appreciated by readers not familiar with matroid theory in advance. These readers are referred to the following section containing a brief introduction to matroid theory, and to an excellent expository paper by Wilson [22].

\section{A Brief INTRODUCTION TO MATROID ThEORY}

A matroid $M$ is a pair of sets: $M=(S, 9)$. The set $S$ is called the ground set of the matroid, and $\mathscr{B}$ is a set of subsets of the ground set, called bases, $B_{1}, B_{2}, \cdots, B_{r}$ with the following two properties: (b1) no base is properly contained in another base; $(b 2)$ if $B_{i}$ and $B_{j}$ are arbitrary bases, and $x_{i} \in B_{i}$, then an element $x_{j} \in B_{j}$ exists, such that $\left(B_{i} \backslash\left\{x_{i}\right\}\right) \cup\left\{x_{j}\right\}$ is an element of $\mathscr{B}$ (i.e., is a base).

The set of base complements, denoted $\mathscr{B}^{*}$, is the set of bases of a matroid $M^{*}=\left(S, \mathscr{T}^{*}\right)$, called the dual matroid of $M$.

Trees (i.e., spanning trees) of graphs and maximal sets of independent columns of a matrix also have the properties $(b 1)$ and (b2). Thus matroid theory is the common generalization of graph theory and linear algebra. Notice, that graphs can be found for which the set of tree complements is not equal to the set of trees of another graph. These are the nonplanar graphs for which no dual graphs exist. In contrast to this the dual of a matroid always exists.

To give an idea of the concept of matroids, the following examples are given.

\section{Example (2.1)}

Let $H$ denote the graph of Fig. 3. If $\mathscr{T}(H)$ denotes the set of trees of $H$, then

$$
\begin{aligned}
& T(H)=\{\{a, c\},\{a, d\},\{a, e\},\{b, c\}, \\
& \{b, d\},\{b, e\},\{c, e\},\{d, e\}\} .
\end{aligned}
$$

It can be shown that $\mathscr{T}(H)$ equals the set of bases of a matroid $M(H)$, with ground set $S=\{a, b, c, d, e\}$, called the circuit matroid (or polygon matroid) of $H$. The dual matroid of $M(H)$ is called the cutset matroid of $H$, and is denoted $M^{*}(H)$. Every subset of a base is called an independent set, e.g., $\{a\},\{b\},\{c\}$, and $\{d\}$ are independent sets. Notice, that the empty set is an independent set. The subsets of the ground set, which are not independent, are said to be dependent, e.g., $\{a, b, c\},\{b, c, d, e\}$, and $\{c, d, e\}$ are dependent sets of $M(H)$. The minimal dependent sets are called the circuits of the matroid, e.g., $\{a, b\}$, $\{b, c, e\}$, and $\{c, d\}$ are circuits of $M(H)$. The circuits of the dual matroid is called the cocircuits (or cutsets) of the matroid, e.g., $\{a, b, e\},\{c, d, e\}$, and $\{a, b, c, d\}$ are cocircuits of $M(H)$. The cardinality, i.e., the number of elements, of a base is called the rank of the matroid (the 


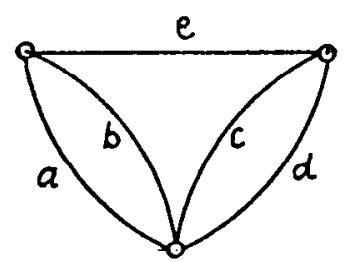

Fig. 3. The graph $H$ of Example (2.1).

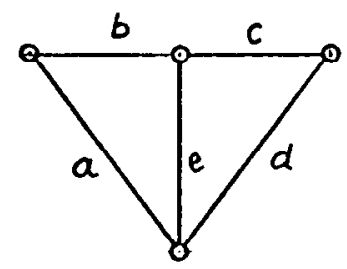

Fig. 4. The dual graph $H^{*}$ of Example (2.1).

bases are equicardinal). The rank of $M(H)$ in this example equals two.

Let $\mathscr{T}^{*}(H)$ denote the set of bases of the dual matroid of $M(H)$. Then

$$
\begin{aligned}
\mathcal{F}^{*}(H)=\{\{b, d, e\}, & \{b, c, e\},\{b, c, d\},\{a, d, e\}, \\
& \{a, c, e\},\{a, c, d\},\{a, b, d\},\{a, b, c\}\}
\end{aligned}
$$

and $M^{*}(H)=(S, \mathcal{T} *(H))$. The set of bases $\mathscr{T}^{*}(H)$, equals the set of trees of the graph $H^{*}$ shown in Fig. 4, i.e., $\mathcal{T}^{*}(H)=\mathcal{T}\left(H^{*}\right)$. Thus $M^{*}(H)=\left(S, \mathcal{T}^{*}(H)\right)=\left(S, \mathcal{T}\left(H^{*}\right)\right)$ $=M\left(H^{*}\right)$, i.e., $M\left(H^{*}\right)$ is the circuit matroid of $H^{*}$ and the cutset matroid of $H$. It may be noticed that $H^{*}$ is the dual of the graph $H$. This is always the case if $H$ is planar.

\section{Example (2.2)}

Let $\{a, b, c, d, e\}$ denote the column set of the matrix $A$ of

$$
A=\left[\begin{array}{ccccc}
a & b & c & d & e \\
3 & 0 & 0 & -2 & 0 \\
9 & 0 & 0 & -6 & 0 \\
6 & 0 & 0 & -4 & 0
\end{array}\right]
$$

If $\delta(A)$ denotes the set of maximal sets of independent columns of $A$, then $\delta(A)=\{\{a\},\{d\}\}$. It can be shown that $\delta(A)$ equals the set of bases of a matroid $M(A)$ with ground set $S=\{a, b, c, d, e\}$, called the matric matroid of $A$. In this example the rank of $M(A)$ equals one.

\section{Example (2.3)}

Two arbitrary matroids are said to be isomorphic, if it is possible to establish a one-to-one correspondance between the ground sets of the matroids, such that a subset, in one of the matroids, is independent iff the corresponding subset is independent in the other matroid.

Let $G$ denote the graph of Fig. 5 and $M(G)$ the circuit matroid of $G$. Then $M(A)$ of Example (2.2), is isomorphic

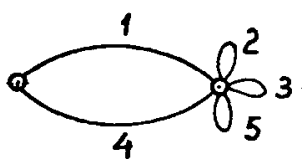

Fig. 5. The graph $G$, the circuit matroid of which is isomorphic to the matric matroid $M(A)$ of Example (2.2).

to $M(G)$, since the following one-to-one correspondence satisfies the condition: $a \leftrightarrow 1, b \leftrightarrow 2, c \leftrightarrow 3, d \leftrightarrow 4, e \leftrightarrow 5$.

If for an arbitrary matroid $M$, a graph $G$ exists such that $M$ is isomorphic to $M(G)$, the matroid $M$ is said to be graphic. Thus the matroid $M(A)$ of Example (2.2) is graphic.

\section{Example (2.4)}

The common ground set of $M(H)$ (Example (2.1)) and $M(A)$ (Example (2.2)) is $S=\{a, b, c, d, e\}$. If we take all possible unions of elements from $\mathscr{T}(H)$ and $\delta(A)$, and denote by $\Re(U)$ the set of unions with maximal cardinality, then,

$$
\begin{aligned}
\mathscr{B}(U)= & \{\{a, b, c\},\{a, b, d\},\{a, b, e\}, \\
& \{a, c, d\},\{a, c, e\},\{a, d, e\}, \\
& \{b, d, e\},\{b, d, e\},\{c, d, e\}\}
\end{aligned}
$$

It can be shown that $\mathscr{B}(U)$ equals the set of bases of a new matroid $M(U)$, with ground set $S$, obtained from $M(H)$ and $M(A)$, in the above way. The matroid $M(U)$ is called the union (sum) of $M(H)$ and $M(A)$, and is denoted $M(U)=(S, \mathscr{B}(U))=M(H) \backslash M(A)$.

In this example $M(U)$ can be shown not to be graphic.

\section{DePendencies Among Voltages AND Currents}

The idea of using graphs in electrical network theory arises from the fact that the basic algebraic Kirchhoff equations are formulated in graph theoretical terms. When no controlled sources are present the dependencies among the voltages are given by the circuits of the graph, and the dependencies among the currents are given by the cutsets of the graph. Since these dependencies are vital for the solvability and the complexity, graphs as a combinatorial tool have been used for many years. But matroids could have been used as well [1], [2]. Instead of the network graph, we could have used the circuit matroid of the graph or the cutset matroid of the graph. (In fact the reader may choose for himself, whether he wants to treat these graphic dependencies as circuits of matroids or as cocircuits (cutsets) of matroids). The main results of this paper are entirely based on consideration of matroid cocircuits, and their relation to certain matrices.

The all-important point in using matroids is that matroid theory combines and generalizes graph theory 
and linear algebra. Matroids are, therefore, the perfect tool for handling, and unifying, the graphic as well as the nongraphic, that is, algebraic dependencies encountered in linear active networks.

\section{The General Case and G-Solvable NETWORKS}

In order to determine if singularity is due to the network topology exclusively, the network parameter values must be eliminated from the treatment. This can be done by assuming all parameters to be general. Generality means that if a specific choice of the network parameter values would cause a network matrix under consideration to become singular, then the parameter values are considered to be different from those specific choices. A more precise definition of generality is given by the following definition.

\section{Definition (4.1)}

An electrical network is $\mathrm{g}$-solvable iff at least one set of network parameter values exists for which the network is solvable.

Since matrices play an important role in the following sections, two more definitions concerning the generality are given.

\section{Definition (4.2)}

A matrix, containing general parameters, is g-nonsingular iff at least one set of parameter values exists for which the matrix is nonsingular (i.e., the determinant is nonzero).

\section{Definition (4.3)}

$A$ set of columns of a matrix, containing general parameters, is g-linearly independent iff at least one set of parameter values exists for which the columns are linearly independent.

Notice, that ordinary nonsingularity (solvability) implies $g$-nonsingularity ( $g$-solvability).

\section{The Matroids OF THE Model}

As mentioned in Section III all the dependencies will be treated by the cocircuits of appropriate matroids.

In the following $S$ denotes the edge set of the network graph $H$. To each element $s \in S$ we associate two other elements $s_{i}$ and $s_{v}$ corresponding to the current and the voltage of the network element, respectively. The two associated sets will be denoted $S_{I}$ and $S_{V}$, i.e., $S_{I}=\left\{s_{i} \mid s \in\right.$ $S\}$ and $S_{V}=\left\{s_{v} \mid s \in S\right\}$. The same notation will be used if subsets of $S, S_{I}$, and $S_{V}$ are considered.

Let $Q_{f}$ denote a fundamental cutset matrix (over the reals) of the directed graph $H$. From graph theory it is well known [20], that a subset of columns of $\boldsymbol{Q}_{f}$ is linearly independent iff the columns correspond to a circuit-free subgraph of $H$. Now the graphic dependencies among the currents due to $\mathrm{KCL}$ will be treated by the following matroid $G_{I}$.

\section{Definition (5.1)}

$G_{I}$ is a matroid on the ground set $S_{I}$. A subset of $S_{I}$ is defined to be independent in $G_{I}$ iff the corresponding columns of $Q_{f}$ are linearly independent.

It is implied by the above description that $G_{I}$ is isomorphic to the circuit matroid $M(H)$. Thus the cocircuits of $G_{I}$ treat $\mathrm{KCL}$.

Similarly, let $\boldsymbol{B}_{f}$ denote a fundamental circuit matrix (over the reals) of the directed graph $H$. Now a subset of columns of $\boldsymbol{B}_{f}$ is linearly independent iff the columns correspond to a cutset-free subgraph of $H$ [20]. The graphic dependencies among the voltages due to KVL will be treated by the following matroid $G_{V}$.

\section{Definition (5.2)}

$G_{V}$ is a matroid on the ground set $S_{V}$. A subset of $S_{V}$ is defined to be independent in $G_{V}$ iff the corresponding columns of $\boldsymbol{B}_{f}$ are linearly independent.

It is implied by the above description that $G_{V}$ is isomor phic to the cutset matroid $M^{*}(H)$, the dual of $M(H)$. Thus the cocircuits of $G_{V}$ treat KVL.

Since the ground sets of $G_{I}$ and $G_{V}$ are disjoint, all graphic dependencies among voltages and currents due to KVL and KCL can be merged by the matroid operation: direct sum. The direct sum of two arbitrary matroids $M_{1}$ and $M_{2}$ on disjoint ground sets $S_{1}$ and $S_{2}$ is denoted $M_{1} \oplus M_{2}$. A set $X$ is independent in $M_{1} \oplus M_{2}$ iff $X \cap S_{1}$ is independent in $M_{1}$ and $X \cap S_{2}$ is independent in $M_{2}$. All the above graphic dependencies will be treated by the following matroid $G$.

$G$ is a matroid on the ground set $S_{I} \cup S_{V}: G=G_{I} \oplus G_{V}$.

In a similar way all nongraphic, that is, algebraic dependencies among voltages and currents, such as control equations, memoryless $n$-port descriptions and resistive element equations may be treated by a matroid.

Let $A$ denote the matrix containing all the above mentioned algebraic equations, such that $A \cdot\left(i_{1}, i_{2}, \cdots, i_{|S|}\right.$, $\left.v_{1}, v_{2}, \cdots, v_{|s|}\right)^{\top}=\underline{0}$. In the general case $A$ contains in each row, precisely one entry equal to 1 which is no network parameter, and the remaining nonzero elements represent different network parameters. Then the algebraic dependencies will be treated by the following matroid $A$.

\section{Definition (5.3)}

$A$ is a matroid on the ground set $S_{I} \cup S_{V}$. A subset of $S_{I} \cup S_{V}$ is defined to be independent in $A$ iff the corresponding columns of $A$ are g-linearly independent.

More specifically, a subset $S^{\prime}$ of $S_{I} \cup S_{V}$ is independent in $A$ iff the matrix formed by the corresponding columns in $A$, contains at least one $g$-nonsingular maximal square submatrix $A^{\prime}$ (see (2)).

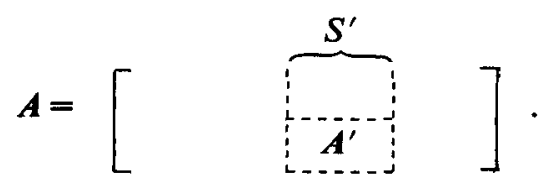


Finally, all information about the dependencies (i.e., Kirchhoff constraints as well as element and $n$-port constraints) in the network can be merged into one single matroid $M$ using the matroid operation: union (or sum). The union of two arbitrary matroids $M_{1}$ and $M_{2}$ on the same ground set $S_{0}$ is denoted $M_{1} \vee M_{2}$. By definition a subset $X \subseteq S_{0}$ is independent in $M_{1} \vee M_{2}$ iff $X=X_{1} \cup X_{2}$ where $X_{1}$ is independent in $M_{1}$ and $X_{2}$ is independent in $M_{2}$. Then,

$M$ is a matroid on the ground set $S_{I} \cup S_{V}: M=G \vee A=\left(G_{I}\right.$ $\left.\oplus G_{V}\right) \bigvee A$.

$M$ is called the network matroid.

To check if a certain set is independent in $M$, it is not necessary to construct the whole set of bases of $M$. It is the advantage of using matroids, that polynomially bounded algorithms are available [8], [10], i.e., algorithms that terminate in at most a number of steps given by a polynomial in the size of the problem (i.e., in this case the number of elements of the ground set). These algorithms [8], [10], check independence in $M$, knowing the independent sets of $G$ and $A$ only. Normally, much storage space in a computer is required to check independence in $G$ and $A$. For the type of problems considered here, however, this can be simplified. The simplification in $G$, is due to the fact, that the network graph $H$ contains all information about $G$. In $A$ the simplification is a consequence of Corollary 6.2 given in the following section. Thus the conditions in the following theorems concerning matroids, can fairly easily be checked.

\section{Networks Without CaPACITORS AND INDUCTORS}

Let $\boldsymbol{N}$ denote the coefficient matrix of the total system of network equations (see (3)).

$$
N\left[\begin{array}{l}
i \\
v
\end{array}\right]=\underline{0}
$$

where

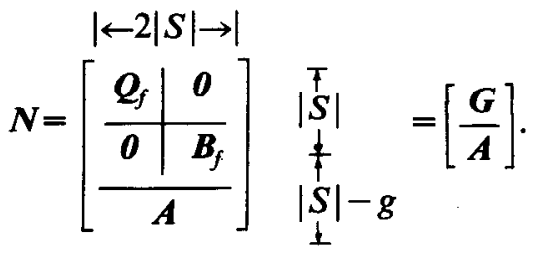

Let $E^{1}$ denote the set of independent voltage sources and $E_{V}^{1}$ denote the corresponding subset of $S_{V}$. Similarly let $I^{1}$ denote the set of independent current sources and $I_{I}^{1}$ denote the corresponding subset of $S_{I}$. Now interchange columns of $N$ to obtain the matrix $N^{\prime}$ (see (4)), where the columns of $N_{1}$ correspond to $E_{V}^{1} \cup I_{I}^{1}$.

$$
N^{\prime}=\left[\begin{array}{l:l}
N_{0} & N_{1}
\end{array}\right]
$$

If $g$ is the number of independent sources then $N_{0}$ consists of $2|S|-g$ rows and columns and $N_{1}$ consists of $2|S|-g$ rows and $g$ columns. Now the network is solvable iff the determinant of $\boldsymbol{N}_{0}$ differs from zero. If the network parameters are assumed to be general, then it is the advantage of using matroids that the determinant need not be calculated. The question of $g$-solvability is answered by the following theorem.

\section{Theorem (6.1)}

Networks without capacitors and inductors are g-solvable iff $E_{V}^{1} \cup I_{I}^{1}$ is a base of $M^{*}$.

Proof: " $\Rightarrow$ ": Expand the determinant of $N_{0}$ by $|S|-g$ columns of $A$, using the Laplace expansion formula. Since det $N_{0} \neq 0$, at least one term in the expansion, e.g., det $\boldsymbol{G}_{0} \cdot \operatorname{det} \boldsymbol{A}_{0}$, is different from zero (see (5)). The columns of $A_{0}$ are linearly independent, and thus the subset of $S_{1} \cup S_{V}$ corresponding to these columns form a base of $A$. Similarly the elements corresponding to the columns of $\boldsymbol{G}_{0}$ form a base of $G$, and since the bases are disjoint, the union is a base of $M$. Thus $E_{V}^{1} \cup I_{I}^{1}$ is a base of $M^{*}$.

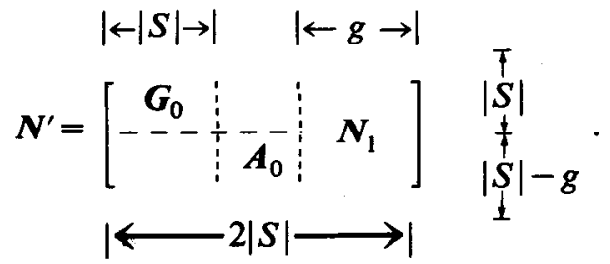

" $\Leftarrow$ ": If $E_{V}^{1} \cup I_{I}^{1}$ is a base of $M^{*}$ then the rank $r_{M}=2|S|$ -g. Since $r_{M} \leqslant r_{G}+r_{A} \leqslant|S|+(|S|-g), r_{M}=r_{G}+r_{A}$. Thus $g$-nonsingular column disjoint submatrices $\boldsymbol{G}_{0}$ and $\boldsymbol{A}_{0}$ exist (see (5)). The determinant of $G_{0}$ equals \pm 1 . Since $A_{0}$ is $g$-nonsingular, at least one set of network parameter values exists, such that $A_{0}$ is nonsingular. Choose the network parameter values of $\boldsymbol{A}_{0}$ to equal such a set, and choose arbitrarily a set of finite values for the remaining network parameters. Now at least one term in the expansion of the determinant of $A_{0}$ differs from zero. Thus a permutation of the columns of $A_{0}$ exists such that the diagonal elements of the resulting matrix are nonzero. Some of these distinguished nonzero elements represent network parameters, others are the nonparameter element 1. Now imagine all distinguished nonzero elements to equal a common variable $x$, while all other elements remain unchanged. The determinant of $N_{0}$ thus is a polynomial in $x$. Furthermore the determinant differs from the zero polynomial, since the coefficient of $x^{(|S|-g)}$ equals $\operatorname{det}\left(G_{0}\right)$, i.e., \pm 1 . Thus an infinite number of finite and nonzero values $x_{0}$ exist, such that the determinant of $N_{0}$ differs from zero. Choose one of these values. If a distinguished nonzero element represents a network parameter, then that parameter assumes the value $x_{0}$. If the distinguished nonzero element is the nonparameter element 1 , the values of the network parameters in that row are divided by $x_{0}$ (i.e., the row is scaled by $x_{0}$ ). Thus a set of network parameter values has been found such that the determinant of $\boldsymbol{N}_{0}$ differs from zero, i.e., the network is $g$-solvable.

Notice that the proof of the necessity did not use the generality at all. More specifically the solvability follows from the fact that, under the conditions of Theorem (6.1) all voltages and currents $y^{\top}$ corresponding to elements 
not in $E_{Y}^{1} \cup I_{I}^{1}$, can be expressed as a unique linear combination of the voltages and currents $\boldsymbol{x}^{\top}$ corresponding to elements of $E_{V}^{1} \cup I_{I}^{1}$ :

$$
y^{\top}=-N_{0}^{-1} N_{1} x^{\top}
$$

The following corollary is an immediate consequence of the proof of Theorem (6.1).

\section{Corollary (6.2)}

Let $A_{0}$ denote a maximal square submatrix of $A$. Then $A_{0}$ is g-nonsingular iff a permutation of the columns of $A_{0}$ can be found such that the resulting matrix has diagonal elements which are nonzero.

Similarly a subset of columns of $\boldsymbol{A}$ are $g$-linearly independent iff the matrix formed by these columns contains at least one maximal square submatrix, for which the desired permutation of columns can be found.

Now consider a bipartite graph, the vertices of which correspond to the rows and the columns of $A$, respectively, and the edges of which correspond to the nonzero elements of $\boldsymbol{A}$. Then the algorithm which finds a maximal matching in a bipartite graph, can be used to find a maximal square submatrix with the desired properties, and a permutation of columns of $\boldsymbol{A}_{0}$. Hence, the test for independence in $A$ is fairly simple.

To give an idea of the matroid $M$, notice that the matroid $M-\left(E_{V}^{1} \cup I_{I}^{1}\right)$ obtained from $M$ by deleting $E_{V}^{1} \cup$

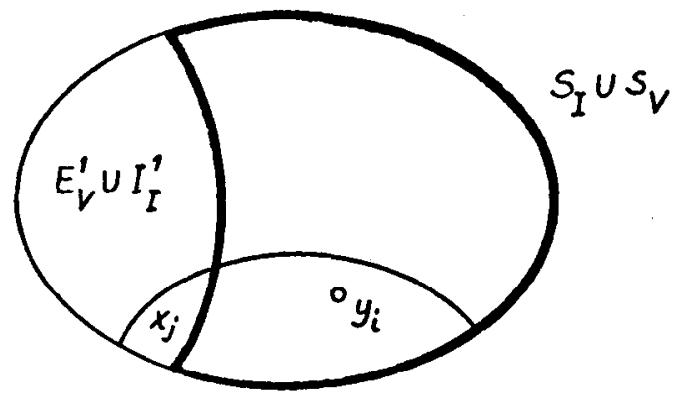

Fig. 6. The area bounded by heavy lines is the base $\left(S_{I} \cup S_{V}\right) \backslash$ $\left(E_{Y}^{\mathrm{Y}} \cup I_{I}^{1}\right)$ of $M$.

The examples of Section I will now be considered more closely. In the following examples the total system of network equations and the associated matroids will be given. If the matroids are graphic, graphs will be given, such that the matroids are the circuit matroids of the graphs. It may be noticed that, if the graph $H$, corresponding to $G_{I}$, is planar, then the graph corresponding to $G_{V}$ is the dual of $H$.

\section{Example (6.3)}

The network $N_{1}$, of Example (1.1), is shown in Fig. 1. The total system of network equations is given in (7). The submatrix $\boldsymbol{N}_{0}$ is formed by the columns corresponding to $1_{i}, 2_{i}, 4_{i}, 5_{i}, 6_{i}, 7_{i}, 3_{v}, 4_{v}, 5_{v}, 6_{v}, 7_{v}$.

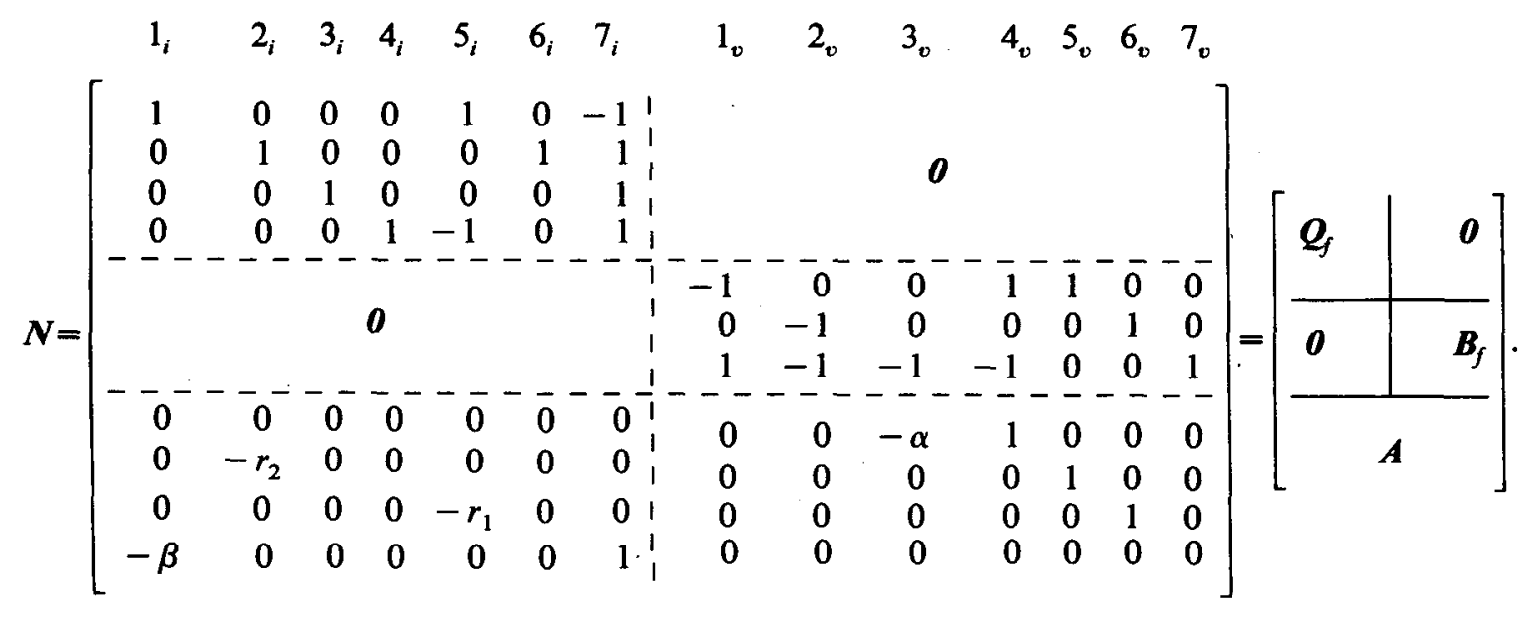

$I_{I}^{1}$, in case of regularity is the free matroid, i.e., $M-\left(E_{V}^{1} \cup\right.$ $\left.I_{I}^{1}\right)$ contains no circuits.

Furthermore, the fundamental circuits of $M$ with respect to the base $\left(S_{I} \cup S_{V}\right) \backslash\left(E_{V}^{1} \cup I_{I}^{1}\right)$ and defined by the elements of the base complement $E_{V}^{1} \cup I_{l}^{1}$, are related to the nonzero elements of $N_{0}^{-1} N_{1}$, in the following way. The element in the $i$ th row and $j$ th column of $N_{0}^{-1} N_{1}$ is nonzero, iff the element corresponding to $y_{i}$ is an element of the fundamental circuit of $M$ with respect to the base $\left(S_{I} \cup S_{V}\right) \backslash\left(E_{V}^{1} \cup I_{I}^{1}\right)$ and defined by the element of the base complement $E_{V}^{1} \cup I_{I}^{1}$ corresponding to $x_{j}$ (see Fig. 6). The elements of these fundamental circuits of $M$ are easily obtained using the algorithm which checks the condition of Theorem (6.1).
The associated matroids are shown in Fig. 7 (in this case the matroids $G_{I}, G_{V}$, and $A$ are graphic).

According to Theorem (6.1), the matrix $N_{0}$ is g-nonsingular iff $E_{V}^{1} \cup I_{I}^{1}=\left\{1_{v}, 2_{v}, 3_{i}\right\}$ is a base of $M^{*}$, i.e., the complementary set $B=\left\{3_{v}, 4_{v}, 5_{v}, 6_{v}, 7_{v}, 1_{i}, 2_{i}, 4_{i}, 5_{i}, 6_{i}\right.$, $7_{i}$ \} is a base of $M$. A reformulation of this condition is, that disjoint bases of $G_{I}, G_{V}$, and $A$ exist, such that the union of these bases equals the complementary set $B$.

To find out whether such disjoint bases exist, $\left\{1_{v}, 2_{v}, 3_{i}\right\}$ is deleted from the matroids (deleted elements are denoted by crossed edges in Fig. 7). Since $\left\{6_{v}, 2_{v}\right\}$ is a cocircuit (cutset) in $G_{V},\left\{6_{v}\right\}$ must be an element of $B$ (the elements forced into $B$, are denoted by heavy edges in Fig. 7). Now, $\left\{6_{v}, 5_{i}\right\}$ is a cocircuit (cutset) of $A$, and $\left\{5_{i}\right\}$ must be an 


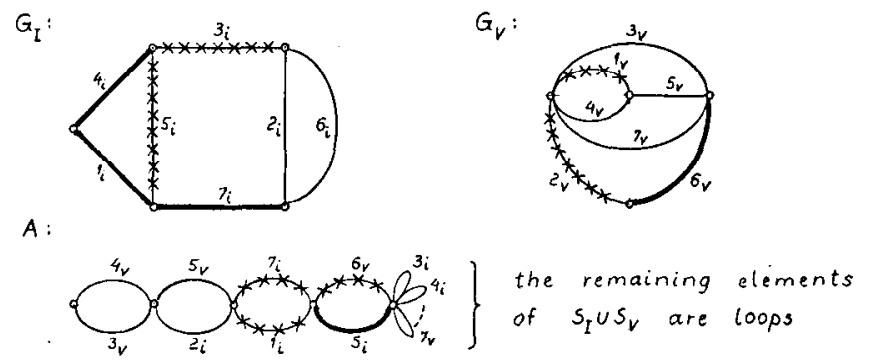

Fig. 7. The matroids $G_{I}, G_{V}$, and $A$ associated with the network $N_{1}$ of Example (6.3) (Fig. 1).

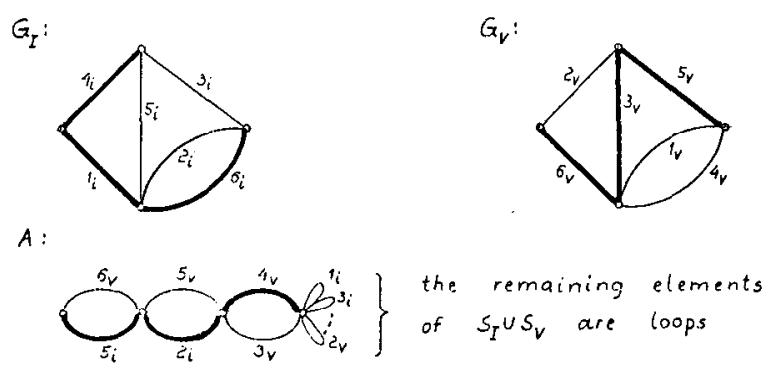

Fig. 8. The matroids $G_{I}, G_{V}$, and $A$ associated with the network $N_{2}$ of Example (6.4) (Fig. 2).

element of $B$. When $\left\{3_{i}, 5_{i}\right\}$ is deleted from $G_{I},\left\{4_{i}, 1_{i}, 7_{i}\right\}$ must be contained in $B$. So the disjoint bases, mentioned in the condition, do not exist, since $\left\{1_{i}, 7_{i}\right\}$ is a cocircuit (cutset) of $A$. Thus, according to Theorem (6.1), the determinant of $N_{0}$ equals zero for any choice of the network parameter values.

\section{Example (6.4)}

The network $N_{2}$, of Example (1.2), is shown in Fig. 2. The associated matroids are given in Fig. 8 (in this case $G_{I}, G_{V}$, and $A$ are graphic).

It is easily checked that $\left\{1_{v}, 2_{v}, 3_{i}\right\}$ is a base of $M^{*}$, i.e., $\left\{1_{i}, 2_{i}, 4_{i}, 5_{i}, 6_{i}, 3_{v}, 4_{v}, 5_{v}, 6_{v}\right\}$ is a base of $M$ (the heavy edges of Fig. 8). Thus $\mathrm{N}_{2}$ is $\mathrm{g}$-solvable.

If specific choices of network parameter values are made, $N_{2}$ may become singular. To find these specific choices, the total system of network equations is considered (8).

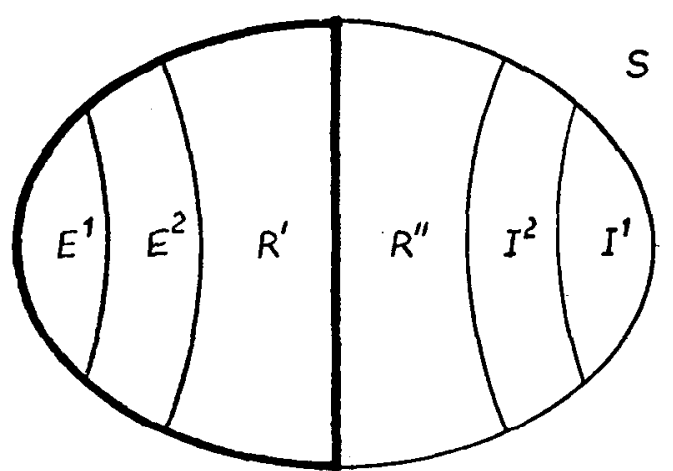

Fig. 9. The area bounded by heavy lines is the tree $\boldsymbol{T}$ of $\boldsymbol{H}$.

equals $r_{1} r_{2}(1+\alpha)$. Thus $N_{2}$ is singular iff $r_{1}=0, r_{2}=0$, or $\alpha=-1$.

Theorem (6.1) has the following interesting corollary.

\section{Corollary (6.5)}

Networks with independent and controlled sources and resistors only, containing no circuits formed by voltage sources alone and no cutsets formed by current sources alone, are g-solvable.

Proof: Let $E^{2}$ and $I^{2}$ denote the set of controlled voltage- and current sources, respectively. If $H$ denotes the network graph, then $E^{1} \cup E^{2}$ contains no circuits of $H$. Similarly, $I^{1} \cup I^{2}$ contains no cutset of $H$. Now, it is well known ([20], Theorem 6-10) that a tree $T$ can be found such that $E^{1} \cup E^{2} \subseteq \top$ and $I^{1} \cup I^{2} \subseteq \top^{*}$, where $\top^{*}$ denotes the tree complement. Since $G_{I}$ is isomorphic to $M(H)$ and $G_{V}$ is isomorphic to $M^{*}(H),(T)_{I}$ and $\left(T^{*}\right)_{V}$ are bases of $G_{I}$ and $G_{V}$, respectively, (see Fig. 9). Thus $(\mathrm{T}), \cup\left(T^{*}\right)_{V}$ is a base of $G$.

Let $R^{\prime}$ and $R^{\prime \prime}$ denote the resistors contained in $T$ and $T^{*}$, respectively. Choose from $A$ the elements $D=E_{V}^{2} \cup$ $\left(R^{\prime}\right)_{V} \cup\left(R^{\prime \prime}\right)_{I} \cup I_{I}^{2}$.

The row of $A$, corresponding to an element of $E^{2} \cup R \cup$ $I^{2}$, contains a nonzero element in the column of $A$, corresponding to that specific element of $D$. Since the cardinality of $D$ equals the number of rows of $A$, and since $|D|$ nonzero elements, in different rows and col-

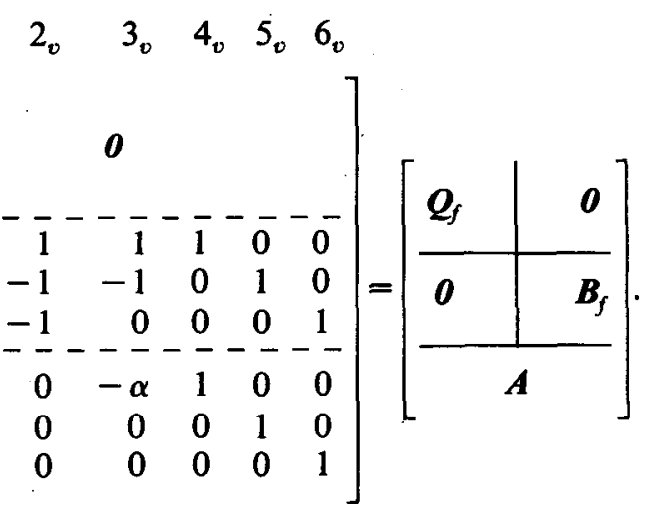

The submatrix $N_{0}$ is formed by the columns corresponding to $i_{1}, i_{2}, i_{4}, i_{5}, i_{6}, v_{3}, v_{4}, v_{5}, v_{6}$. The determinant of $N_{0}$ umns, have been found, $D$ is a base of $A$ (according to Corollary (6.2)). Since $D$ and $T_{I} \cup\left(T^{*}\right)_{V}$ are disjoint, 
$D \cup T_{I} \cup\left(T^{*}\right)_{V}$ is a base of $M$, and thus $E_{V}^{1} \cup I_{I}^{1}$ is a base of $M^{*}$. Hence, according to Theorem (6.1), the network is g-solvable.

Sufficient conditions, very much similar to those of Corollary (6.5), have been given by Ozawa [14, theorem 2].

The conditions of Corollary (6.5) are sufficient only. Example (1.2) gives a counterexample to the necessity. However, necessary conditions for solvability, have been given by Purslow [17]. The conditions of Purslow allow circuits formed by voltage sources, if the current in at least one of the elements of the circuit is a controlling quantity somewhere in the network. Similarly cutsets formed by current sources are allowed, if the voltage in at least one of the elements of the cutset is a controlling quantity. The conditions of Purslow are necessary only, which is shown in Example (1.1).

Sufficient conditions, similar to those of Corollary (6.5) can be given, if arbitrary memoryless $n$-ports are allowed in the network.

\section{N-PORT DESCRIPTIONS}

From the networks treated in Section VI, the memoryless $n$-ports naturally arise, in the following way. Point out $n$ arbitrary pairs of vertices, which will be considered as the ports of the $n$-port. Then insert an edge for each port.

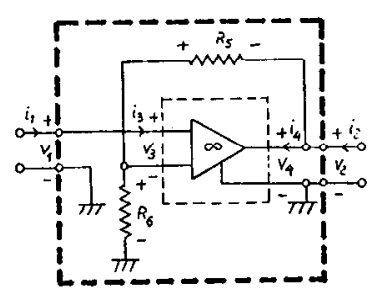

Fig. 10. The 2-port $N_{3}$ of Example (7.1).

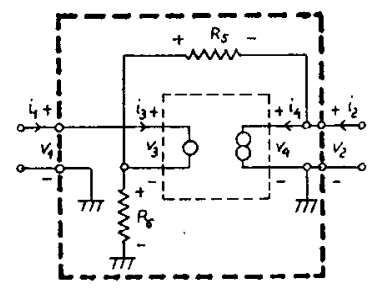

Fig. 11. The equivalent network $N_{3}^{\prime}$ of Example (7.1).

fier, and the equivalent network is shown in Fig. 11. The description of the norator-nullator pair is given in (9).

$$
\left[\begin{array}{l}
v_{3} \\
i_{3}
\end{array}\right]=\left[\begin{array}{ll}
0 & 0 \\
0 & 0
\end{array}\right]\left[\begin{array}{l}
v_{4} \\
i_{4}
\end{array}\right]
$$

The total system of the network equations is given in (10).

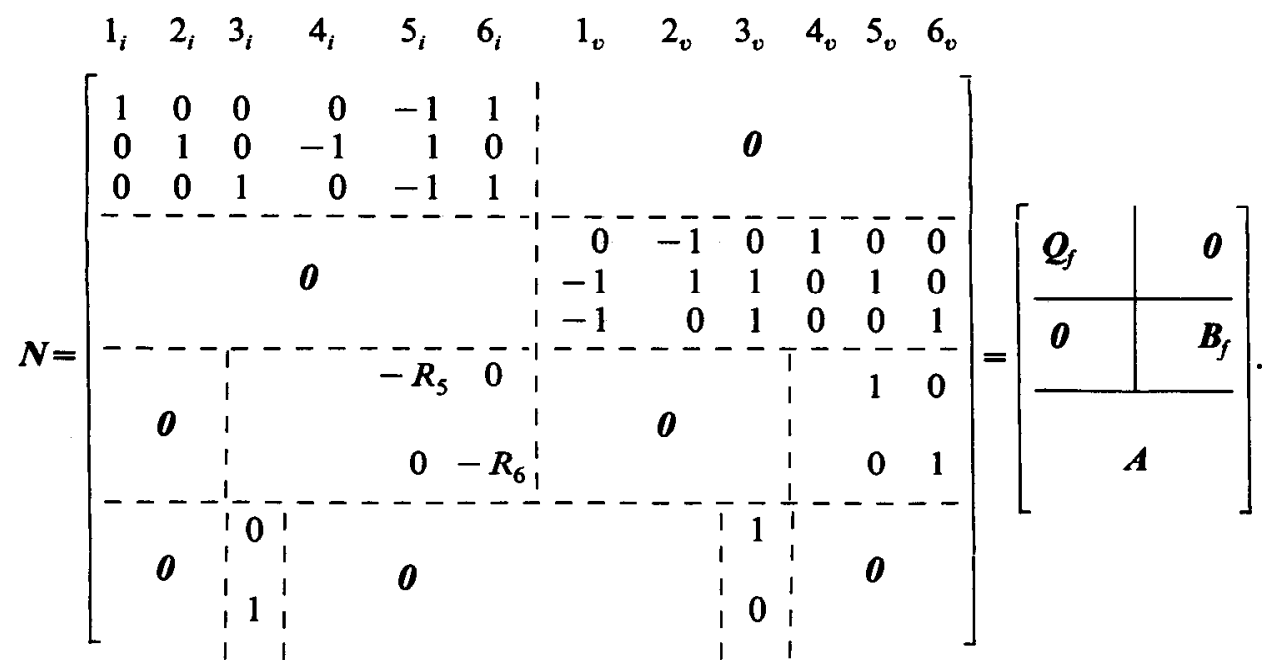

These edges are called port-edges, and the set of port edges is denoted by $\boldsymbol{P}$. If the network contains independent sources, then for each source, the pair of vertices incident to the source are considered to be one of the $n$-ports.

Let $\boldsymbol{x}$ denote a $n$-tuple containing port voltages and/or port currents, and $y$ denote another $n$-tuple containing the remaining port voltages and port currents. Then $y=N x$ is called a matrix description of the $n$-port.

\section{Example (7.1)}

A 2-port $N_{3}$ is given in Fig. 10.

The norator-nullator equivalent (considered here as a 2-port within $N_{3}$ ) is used for the ideal operational ampli-
A possible description of the 2-port is given in (11).

$$
\left[\begin{array}{l}
i_{1} \\
v_{2}
\end{array}\right]=\left[\begin{array}{cc}
0 & 0 \\
\frac{R_{5}}{R_{6}}+1 & 0
\end{array}\right]\left[\begin{array}{c}
v_{1} \\
i_{2}
\end{array}\right] .
$$

The question of interest, is to find the types of matrix descriptions, if any at all. This question is answered by the following theorem.

\section{Theorem (7.2)}

There exists at least one description iff there exist subsets $P^{1}, P^{2}$ of $P$ such that $\left|P^{1}\right|+\left|P^{2}\right|=|P|$ and $P_{V}^{1} \cup P_{I}^{2}$ is a base of $M^{*}$. 
$G_{I}$

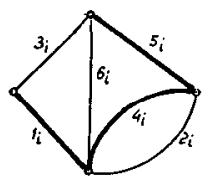

$G_{v}:$

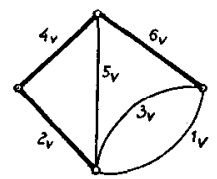

A :

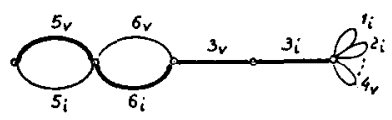

Fig. 12. The matroids $G_{l}, G_{Y}$, and $A$ associated with the 2-port $N_{3}$ of Example (7.1) (Fig. 10 or Fig. 11).

If a description exists, the elements of $P_{V}^{1} \cup P_{I}^{2}$ correspond to the elements of $x$.

The theorem can easily be proved, following the ideas in the proof of Theorem (6.1). The only thing which has to be noticed is that $P^{1}$ and $P^{2}$ need not be disjoint. If they are disjoint, $P_{V}^{1} \cup P_{I}^{2}$ is said to be $I-V$-disjoint, and $y=N x$ is called a hybrid immittance description (see, e.g., the description (11)).

The well-known open circuit impedance description and the short circuit admittance description, are both hybrid immittance descriptions. Theorem (7.2) is illustrated by the following example.

\section{Example (7.3)}

The 2-port of this example is the 2-port of Example (7.1), shown in Fig. 10. The matroids of the 2-port is shown in Fig. 12. It is easily verified that $\left\{1_{v}, 2_{i}\right\}$ and $\left\{2_{v}, 2_{i}\right\}$ are bases of $M^{*}\left(\left(S_{I} \cup S_{V}\right) \backslash\left\{1_{v}, 2_{i}\right\}\right.$ is a base of $M$, which is illustrated by the heavy edges of Fig. 12). The set $\left\{1_{v}, 2_{i}\right\}$ corresponds to the description (11) and the set $\left\{2_{v}, 2_{i}\right\}$ corresponds to the description (12). Since a 2-port is considered, the description (12) is called a chain description.

$$
\left[\begin{array}{c}
v_{1} \\
i_{1}
\end{array}\right]=\left[\begin{array}{cc}
\frac{R_{6}}{R_{5}+R_{6}} & 0 \\
0 & 0
\end{array}\right]\left[\begin{array}{c}
v_{2} \\
-i_{2}
\end{array}\right] .
$$

The zero entries of the descriptions (11) and (12) could have been predicted, since none of the sets $\left\{1_{v}, 1_{i}\right\}$, $\left\{1_{v}, 2_{v}\right\},\left\{1_{i}, 2_{i}\right\}$, and $\left\{1_{i}, 2_{v}\right\}$ are bases of $M^{*}$. If, for example, the element of the second row and the first column in the description (12) had been nonzero, a description with $x=\left(i_{1}, i_{2}\right)$ would have existed. Thus $\left\{1_{i}, 2_{i}\right\}$ would have been a base of $M^{*}$, which however, is not the case.

Furthermore, the element in the $i$ th row and $j$ th column of a description is nonzero (in the general case) iff the element corresponding to $y_{i}$ is an element of the fundamental circuit of $M$ with respect to the base $\left(S_{I} \cup S_{V}\right) \backslash\left(P_{V}^{1}\right.$ $\cup P_{I}^{2}$ ) and defined by the element of the base complement $P_{V}^{1} \cup P_{I}^{2}$ corresponding to $x_{j}$ (see Fig. 2). Theorem (7.2) has the following corollary.

\section{Corollary (7.4)}

There exists at least one hybrid immittance description iff there exist subsets $P^{1}, P^{2}$ of $P$ such that $P^{1} \cap P^{2}=\varnothing$, $P^{1} \cup P^{2}=P$, and $P_{V}^{1} \cup P_{I}^{2}$ is a base of $M^{*}$.
If a description exists, the elements of $P_{V}^{1} \cup P_{I}^{2}$ correspond to the elements of $\boldsymbol{x}$.

Corollary (7.4) answers the following interesting questions. Is it possible to attach independent sources to the ports, such that the obtained network is $g$-solvable? If it is possible, how can it be done? Thus in Example (7.1) the only possible set of independent sources is a voltage source at port 1 and a current source at port 2 , if the obtained network is to be $g$-solvable.

\section{Networks CONTAINING CAPACITORS AND INDUCTORS}

The results and ideas of Section VI, now enable us to solve the interesting problem concerning the complexity of the network.

The following theorem gives the exact number in the general case, in the following denoted g-complexity, and an upper bound if specific choices of network parameter values are taken into consideration.

In the following $L$ and $C$ denote the set of inductors and capacitors, respectively. Subsets of $L$ and $C$ are denoted $L^{0}$ and $C^{0}$, respectively.

\section{Theorem (8.1)}

If $\left|C_{Y}^{0} \cup L_{I}^{0}\right|$ is maximum with respect to $E_{V}^{1} \cup C_{V}^{0} \cup(L)$ $\left.L^{0}\right)_{V} \cup\left(C \backslash C^{0}\right)_{I} \cup L_{I}^{0} \cup I_{I}^{1}$ being a base of $M^{*}$ then $\mid C_{V}^{0} \cup$ $L_{I}^{0} \mid$ is the g-complexity of the network, and the capacitor voltages and inductor currents corresponding to the elements of $C_{V}^{0} \cup L_{J}^{0}$ form a set of independent state variables.

Proof: First the statement on the complexity is proved. Let the number of reactive elements (i.e., inductors and capacitors) be $k$, and put the total system of network equations in the form of (13):

$$
\left[\begin{array}{c}
N \\
\hdashline \boldsymbol{Z}
\end{array}\right] \cdot\left[\begin{array}{l}
i \\
v
\end{array}\right]=T \cdot\left[\begin{array}{l}
i \\
v
\end{array}\right]=0
$$

such that $\boldsymbol{Z}$ represents the Laplace transformed element descriptions of the inductors and capacitors (see (14)) where $\boldsymbol{L}$ and $\boldsymbol{C}$ represent diagonal matrices and $\boldsymbol{U}$ represents a unit matrix).

$$
\begin{aligned}
& 1 \longleftrightarrow-2|S \longmapsto| \\
& \left(C \backslash C^{0}\right)_{I} L_{I}^{0} \quad C_{V}^{0} \quad\left(L \backslash L^{0}\right)_{V}
\end{aligned}
$$

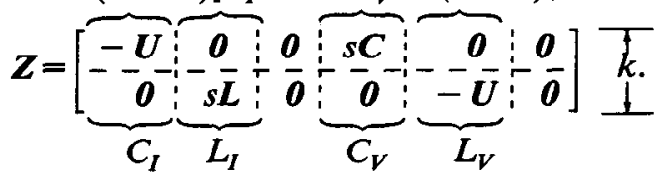

Now interchange columns of $\boldsymbol{T}$ to obtain the matrix $\boldsymbol{T}^{\prime}$ (see (15)), where the columns of $T_{1}$ correspond to $E_{V}^{1} \cup I_{I}^{1}$. The columns of $T_{0}$ form a $(2|S|-g) \times(2|S|-g)$ matrix. The determinant of $T_{0}$ is a polynomial in $s$, the degree of which is the complexity of the network.

$$
T^{\prime}=\left[\begin{array}{l:l}
T_{0} & T_{1}
\end{array}\right]=\left[\begin{array}{c:c}
N_{0} & \\
\hdashline Z_{0} & T_{1}
\end{array}\right]
$$

The determinant of $\boldsymbol{T}_{0}$ is now expanded using the Laplace 
expansion formula. The determinant of the matrices $Z_{0}^{\prime}$ formed by $k$ columns of $Z_{0}$ (see (16)) is different from zero iff the corresponding subset of $S_{I} \cup S_{V}$ is $I-V$ disjoint, i.e., of the form $C_{V}^{0} \cup\left(L \backslash L^{0}\right)_{V} \cup\left(C \backslash C^{9}\right)_{I} \cup L_{I}^{0} \subset C_{V}$ $\cup L_{V} \cup C_{l} \cup L_{I}$. The determinant of the matrices $N_{0}^{\prime}$ formed by $2|S|-(k+g)$ columns of $N_{0}$ (see (16)) is different from zero iff the columns correspond to a base of $M$.

$$
\begin{gathered}
2|S|-(k+g) \\
\left.T_{0}=\left[\begin{array}{ccc}
N_{0}^{\prime} & \\
\hdashline & \\
\hdashline & Z_{0}^{\prime}
\end{array}\right]\right]_{\substack{k \\
\downarrow}}
\end{gathered}
$$

The "only if" is due to the generality (Theorem (6.1)). Thus if we assume that the set $E_{V}^{1} \cup C_{V}^{0} \cup\left(L \backslash L^{0}\right)_{V} \cup(C)$ $C^{0}{ }_{I} \cup L_{I}^{0} \cup I_{I}^{1}$ is a base of $M^{*}$ then at least one term in the coefficient of $s^{\left|C_{i}^{0} \cup L_{i}^{0}\right|}$ is different from zero, and since other nonzero terms will contain at least one capacitive or inductive element value corresponding to an element not in $C_{V}^{0} \cup L_{I}^{0}$, the coefficient is, in the general case, different from zero. If, at the same time, $\left|C_{V}^{0} \cup L_{I}^{0}\right|$ is maximum among the subsets of $C_{V} \cup L_{I}$ satisfying the above assumption, then $\left|C_{V}^{0} \cup L_{I}^{0}\right|$ equals the degree of the polynomial in $s$. Hence, $\left|C_{V}^{0} \cup L_{I}^{0}\right|$ is the $g$-complexity of the network.

Next, the statement on the state variables is proved. For each base $X$ of $M^{*}$, the voltages and currents corresponding to elements not in $X$, can be expressed as a linear combination of the voltages and currents corresponding to elements of $X$. Let us consider the elements of $C_{I}^{0} \cup(L)$ $\left.L^{0}\right)_{I} \cup\left(C \backslash C^{0}\right)_{V} \cup L_{V}^{0}$, only. The corresponding voltages and currents can be expressed as shown in (17).

$$
\left[\begin{array}{c}
y_{0} \\
\hdashline y_{1}
\end{array}\right]=\left[\begin{array}{c:c}
F_{11} & F_{12} \\
\hdashline F_{21} & F_{22}
\end{array}\right]\left[\begin{array}{c}
x_{0} \\
\hdashline x_{1}
\end{array}\right]+\left[\begin{array}{c}
H_{10} \\
\hdashline H_{20}
\end{array}\right] u
$$

where $x_{0}$ is a vector formed by the voltages and currents corresponding to the elements of $C_{V}^{0} \cup L_{I}^{0}$, denoted $x_{0} \sim$ $C_{V}^{0} \cup L_{l}^{0}$.

$$
\begin{array}{ll}
x_{1} \sim\left(C \backslash C^{0}\right)_{I} \cup\left(L \backslash L^{0}\right)_{V} & y_{0} \sim C_{I}^{0} \cup L_{V}^{0} \\
y_{1} \sim\left(C \backslash C^{0}\right)_{V} \cup\left(L \backslash L^{0}\right)_{I} & u \sim E_{V}^{1} \cup I_{I}^{1} .
\end{array}
$$

Since $\left|C_{V}^{0} \cup L_{I}^{0}\right|$ is maximum, every symmetric minor of $\boldsymbol{F}_{22}$ is singular. Otherwise, the vector $x_{0}$ could have been expanded by elements of $y_{1}$, contradicting the maximality of $\left|C_{V}^{0} \cup L_{l}^{0}\right|$. (This observation is due to Recski). In particular $\boldsymbol{F}_{22}$ is singular. Thus it is possible by row operations in $F_{21}, F_{22}$, and $\boldsymbol{H}_{20}$, to make the last row of $\boldsymbol{F}_{22}$ consist of zeros only. So the last coordinate $y_{1 n}$ of $y_{1}$, can be expressed as a linear combination of the coordinates of $x_{0}$ and $u$, in the following denoted by $y_{1 n}=L_{1}\left(x_{0}, u\right)$. Now the derivative of $y_{1 n}$ is proportional to $x_{1 n}$, and at the same time it can be expressed as a linear combination of the elements of $x_{0}, x_{1}, u$, and $\dot{u}: k x_{1 n}=L_{2}\left(\dot{x}_{0}, \dot{u}\right)=$ $L_{3}\left(x_{0}, x_{1}, u, \dot{u}\right)$. The last equality is due to the fact that the derivative of each coordinate of $x_{0}$ is proportional to the same coordinate of $y_{0}$. Since the network parameters are general $x_{1 n}$ can be expressed as a linearcombination of the coordinates of $x_{0}, x_{1}^{\prime}, u$, and $\dot{u}$, where $\left(x_{1}^{\prime}, x_{1 n}\right)=x_{1}: x_{1 n}=$ $L_{4}\left(x_{0}, x_{1}^{\prime}, u, \dot{u}\right)$. A new system of equations is then obtained (see the following):

$$
\left[\begin{array}{c}
y_{0} \\
\hdashline y_{1}^{\prime}
\end{array}\right]=\left[\begin{array}{c:c}
F_{11}^{\prime} & F_{12}^{\prime} \\
\hdashline F_{21}^{\prime} & F_{22}^{\prime}
\end{array}\right]\left[\begin{array}{c}
x_{0} \\
\hdashline x_{1}^{\prime}
\end{array}\right]+\left[\begin{array}{c}
H_{10}^{\prime} \\
\hdashline H_{20}^{\prime}
\end{array}\right] u+\left[\begin{array}{c}
H_{11} \\
\hdashline H_{21}
\end{array}\right] \dot{u}
$$

Every symmetric minor of $F_{22}^{\prime}$ is still singular and the process can be repeated. Finally the system of (19) is obtained.

$$
\boldsymbol{y}_{0}=\boldsymbol{F}_{11}^{n} \boldsymbol{x}_{0}+\sum_{j=0}^{n} \boldsymbol{H}_{j}^{(j)}
$$

Since $y_{0}=D \dot{x}_{0}$, where $D$ is a diagonal matrix, the state equations are obtained (see the following):

$$
D \dot{x}_{0}=y_{0}=F_{11}^{n} x_{0}+\sum_{j=0}^{n} H_{j}^{(j)}
$$

or

$$
\dot{x}_{0}=D^{-1} F_{11}^{n} x_{0}+D^{-1} \sum_{j=0}^{n} H_{j}^{(j)} .
$$

The set of algebraic equations, obtained during the process, is a part of the system of equations expressing all remaining voltages and currents as linear combinations of the coordinates of $x_{0}$ and $(j)$.

It appears from the proof of Theorem (8.1) that $\max \mid C_{V}^{0}$ $\cup L_{I}^{0} \mid$ is an upper bound on the complexity, i.e., $\sigma \leqslant \sigma_{g}=$ $\max \left|C_{V}^{0} \cup L_{I}^{0}\right|$. The upper bound is reached if the topology alone is taken into consideration. Specific choices of the network parameter values may decrease the complexity.

If $\left|C_{V}^{0} \cup L_{I}^{0}\right|$ is minimum, instead of maximum, then a lower bound on the number of dc-eigenfrequencies $\delta$ is found, i.e., $\delta>\delta_{g}=\min \left|C_{V}^{0} \cup L_{I}^{0}\right|$. Furthermore, the qualitative appearance of the determinant, considered as a polynomial in $s$, can in the general case be found.

The proof of Theorem (8.1) has the following immediate corollary.

\section{Corollary (8.2)}

$R L C$ networks containing controlled sources are g-solvable iff subsets $C^{0} \subseteq C$ and $L^{0} \subseteq L$ exist such that $E_{V}^{1} \cup C_{V}^{0}$ $\cup\left(L \backslash L^{9}\right)_{V} \cup\left(C \backslash C^{9}\right)_{I} \cup L_{I}^{0} \cup I_{I}^{1}$ is a base of $M^{*}$.

A corollary similar to Corollary (6.5) can be given, if capacitors and inductors are allowed in the network.

\section{Corollary (8.3)}

$R L C$ networks containing controlled sources are g-solvable if the set $E^{1} \cup E^{2}$ does not contain any circuit, and the set $I^{1} \cup I^{2}$ does not contain any cutset of the network graph.

The theorems concerning the complexity are now illustrated by some examples. 


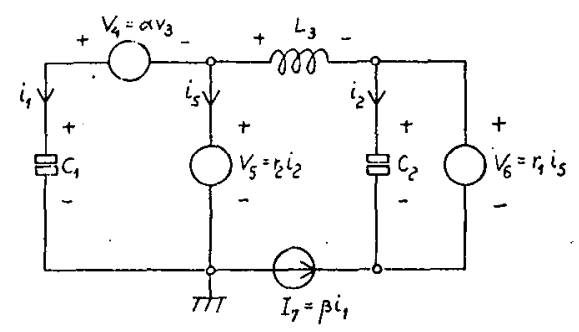

Fig. 13. The network $N_{4}$ of Example (8.4).

$G_{I}:$
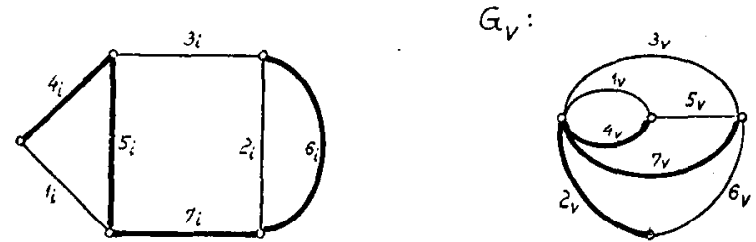

$A:$

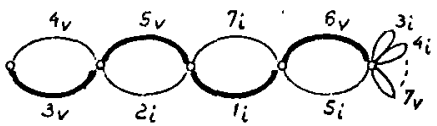

Fig. 14. The matroids $G_{f}, G_{V}$, and $A$ associated with the network $N_{4}$ of Example (8.4) (Fig. 13).

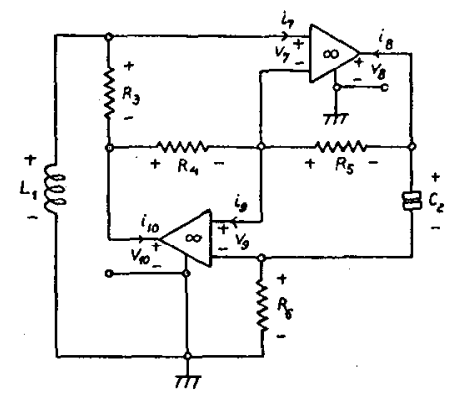

Fig. 15. The network $N_{5}$ of Example (8.5).
$\boldsymbol{G}_{I}$

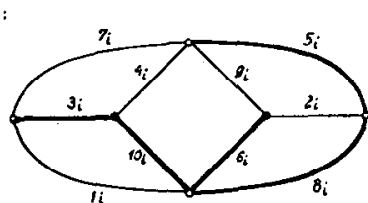

$\mathbf{G}_{\boldsymbol{v}}$ :

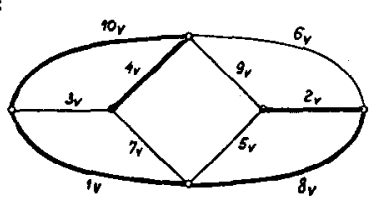

$A$ :

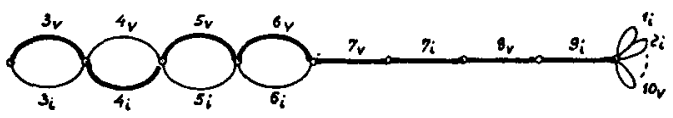

Fig. 16. The matroids $G_{f}, G_{V}$, and $A$ associated with the network $N_{5}$ or Example (8.5) (Fig. 15).

\section{Example (8.4)}

The network $N_{4}$ is shown in Fig. 13. The total system of network equations is shown in (21).

are taken into account the complexity may decrease. For instance, if $C_{2} / L_{3}=r_{1} r_{2} / \alpha$ the complexity equals zero.

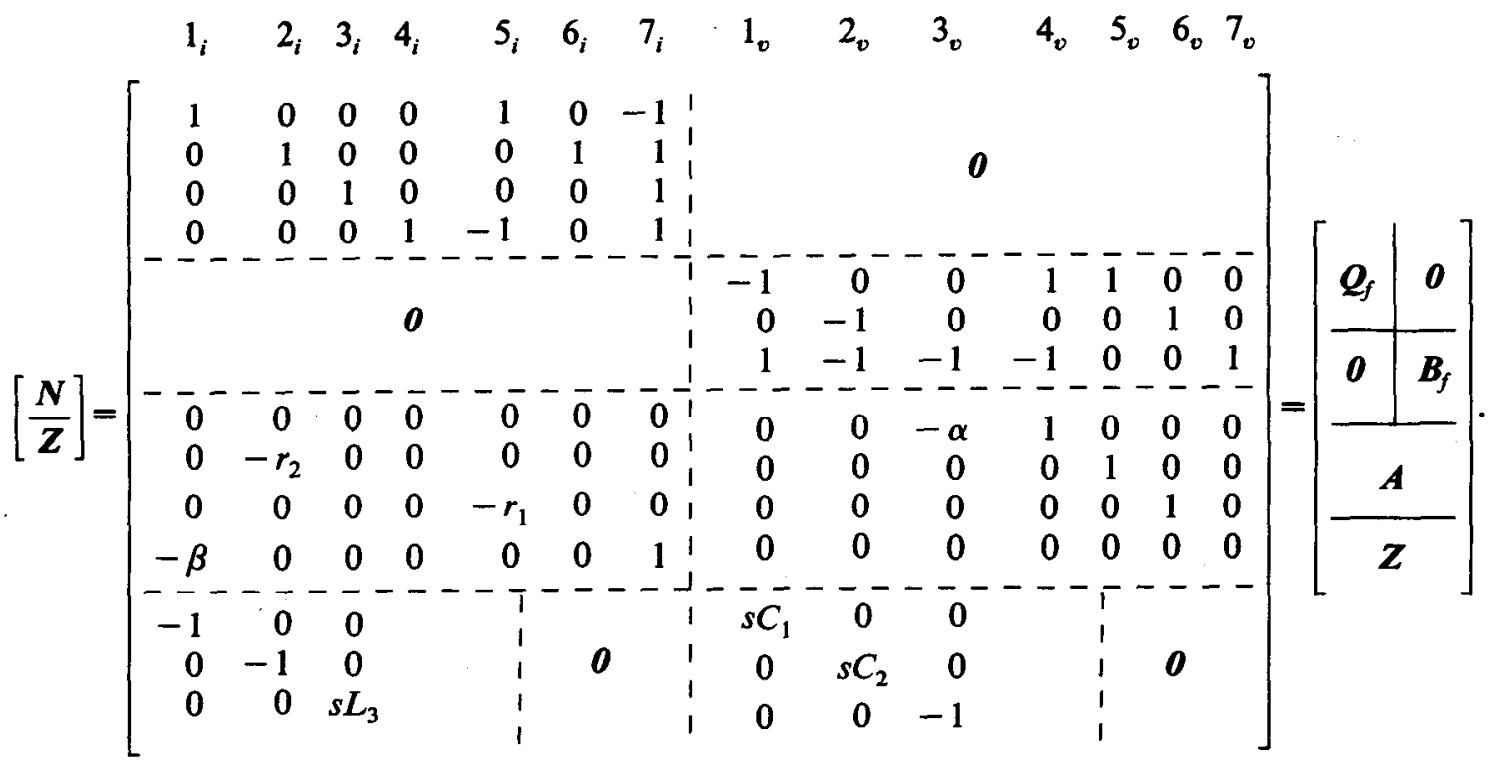

The associated matroids are given in Fig. 14 (in this case graphic). It can be verified that $\left\{1_{v}, 2_{i}, 3_{i}\right\}$ and $\left\{1_{v}, 2_{v}, 3_{v}\right\}$ are bases of $M^{*}$ and that $\left\{1_{v}, 3_{i}\right\}$ and $\left\{1_{v}, 2_{v}\right\}$ are maximum sets. $\left(\left(S_{I} \cup S_{V}\right) \backslash\left\{1_{v}, 2_{i}, 3_{i}\right\}\right.$ is a base of $M$, which is illustrated by the heavy edges of Fig. 14). Thus the g-complexity is two. If specific choices of network parameters

\section{Example (8.5)}

The network $N_{5}$ is shown in Fig. 15. The matroids of the network is shown in Fig. 16.

It can easily be verified that $\left\{1_{i}, 2_{i}\right\}$ and $\left\{1_{v}, 2_{v}\right\}$ are bases of $M^{*}\left(\left(S_{I} \cup S_{V}\right) \backslash\left\{1_{i}, 2_{l}\right\}\right.$ is a base of $M$, which is illustrated by the heavy edges of Fig. 16). Furthermore 


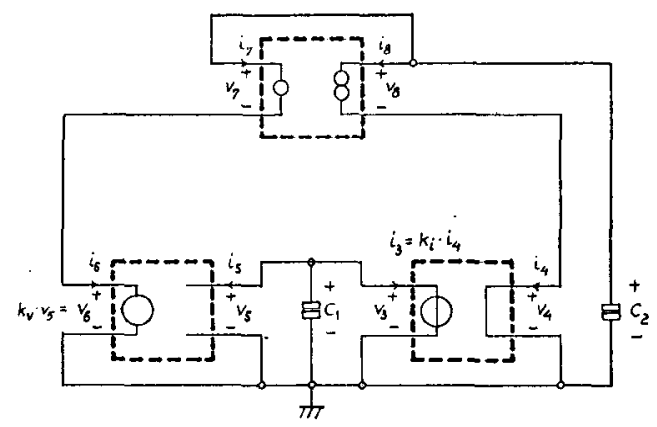

Fig. 17. The network $N_{6}$ of Example (8.6).

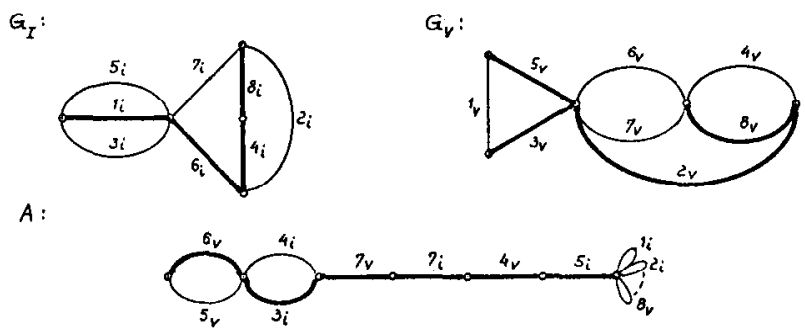

Fig. 18. The matroids $G_{l}, G_{V}$, and $A$ associated with the network $N_{6}$ of Example (8.6) (Fig. 17).

both are maximum and minimum sets. Thus either the complexity and the number of dc-eigenfrequencies both equal one, or the network is singular. Now the network determinant is found to equal $-s\left(C_{2} R_{3} R_{5} R_{6}+L_{1} R_{4}\right)$. Thus if $R_{j}>0(j=3,4,5,6)$ the network is solvable.

\section{Example (8.6)}

The network $N_{6}$ is shown in Fig. 17. The matroids of the network is shown in Fig. 18.

It is easily verified that $\left\{1_{v}, 2_{i}\right\}$ and $\left\{1_{i}, 2_{v}\right\}$ are bases of $M^{*}$, and $\left\{1_{v}\right\}$ or $\left\{2_{v}\right\}$ are maximum sets $\left(\left(S_{I} \cup S_{\nu}\right)\right\}$ $\left\{1_{v}, 2_{i}\right\}$ is a base of $M$, which is illustrated by the heavy edges of Fig. 18). Thus the $g$-complexity is one. Furthermore $\left\{1_{v}\right\}$ or $\left\{2_{v}\right\}$ are minimum sets as well. Thus either the complexity and the number of dc-eigenfrequencies both equal one, or the network is singular. The latter occurs if $k_{i} k_{v}=C_{1} / C_{2}$ (in this case, the currents $i_{3}$ and $i_{4}$ are not unique.)

\section{Conclusion}

In the general case, necessary and sufficient conditions for unique solvability of linear active networks have been given. Pure graph theoretical sufficient conditions have also been found. Necessary and sufficient conditions for the existence of $n$-port descriptions were given. A set of independent state variables has been found, and the complexity as well as the number of dc-eigenfrequencies were given.

If the network parameter values are taken into consideration, the necessary and sufficient conditions of the general case, are necessary only. If these conditions are met, then degeneracies are caused by special relations among the network parameter values. Numerical calculations are then unavoidable. The combinatorial tool is matroids. The conditions of the theorems can be checked using polynomially bounded algorithms. A detailed description of the algorithms will be given in a forthcoming paper. The algorithms have been implemented in Fortran IV on an IBM 370/165 computer (e.g., the execution time of Example (6.3) is $0.007 \mathrm{~s}$, and the execution time of Example (8.5) is $0.020 \mathrm{~s}$ ).

\section{ACKNOWLEDGMENT}

The author wishes to thank A. Recski for very fruitful discussions and correspondences. Also very useful discussions with E. V. Sørensen, T. Rübner-Petersen, T. Høholdt, and F. Nielsen, especially during the writing of this paper, are gratefully acknowledged.

\section{REFERENCES}

[1] J. Bruno and L. Weinberg, "Generalized networks: Networks embedded on a matroid, Part 1," Networks vol. 6, pp. 53-94, 1976.

[2] - "Generalized networks: Networks embedded on a matroid Part 2," Networks, vol. 6, pp. 231-272, 1976.

[3] P. R. Bryant, "The explicit form of Bashkow's A-matrix," IRE Trans. Circuit Theory, vol. CT-9, pp. 303-306, Sept. 1962.

[4] W. K. Chen and F. N. T. Chan, "On the unique solvability of linear active networks," IEEE Trans. Circuit Theory, vol. CAS-21, 26-35, Jan. 1974.

[5] A. Dervisoglu, "State equations and initial values in active $R L C$ networks," IEEE Ttans. Circuit Theory, vol. CT-18, Sept. 1971.

[6] K. Hirano, F. Nishi, and S. I. Tomiyama, "Some considerations on state equations of linear active networks,". Circ. Th and Appl., vol. 2, pp. 39-50, 1974.

[7] $\mathbf{M}$. Iri and $\mathbf{N}$. Tomizawa, "A unifying approach to fundamental problems in network theory by means of matroids," Electronics and Communications in Japan, vol. 58-A no. 1, pp. 28-35, 1975. 
[8] D. E. Knuth, "Matroid partitioning," Report, Stan-CS-73-342, Stanford Univ., 1973.

[9] E. S. Kuh and R. A. Rohrer, "The state variable approach to network analysis," Proc. IEEE, vol. 53, pp. 672-686, July 1965.

[10] E. L. Lawler, "Matroid intersection algorithms," Math. Progr., vol. 9, pp. 31-56, 1975.

[11] S. K. Mark and M. N. S. Swamy, "The generalized tree for state variables in linear active networks," Circ. Th. and Appl., vol. 4, pp. 87-92, 1976

[12] W. Mayeda, Graph Theory. New York: Wiley, 1972.

[13] G. J. Minty, "On the axiomatic foundations of the theories of directed linear graphs, electrical networks and network-programming," J. Math. Mech., vol. 15, no. 3, 1966.

[14] T. Ozawa, "Topological conditions for the solvability of active linear networks," Circuit Theory and Appl., vol. 4, pp. 125-136, 1976.

[15] B. Petersen, "Electrical networks and matroids," Ph.D. dissertation, Technical University of Denmark, Lyngby, 1976.

[16] _-, "Circuits in the union of matroids. An algorithmic approach," 1978 (to be submitted).

[17] E. J. Purslow, "Solvability and analysis of linear active networks by use of the state equations," IEEE Trans. Circuit Theory, vol. CT-17, Nov. 1970.

[18] A. Recski, "On partitional matroids with applications," Coll. Math. Soc. J. Bobyai, vol. 10, Infinite and finite sets, Keszthely (Hungary), 1973, North-Holland-American Elsevier, Amsterdam, New York, 1974, pp. 1169-1179.

[19] $\frac{19}{\text {, }}$ "Matroids and independent state variables," presented at Proc. Second European Conf. Circuit Theory and Design, Genova, 1976.
[20] S. Seshu and M. B. Reed, Linear Graphs and Electrical Networks. Reading, MA: Addison-Wesley, 1961.

[21] J. Tow, "Order of complexity of linear active networks," Proc. IEE, vol. 115, pp. 1259-1262, Sept. 1968.

[22] R. J. Wilson, "An introduction to matroid theory," Amer. Math. Monthly, vol. 80 no. 5, pp. 500-525, May 1973.

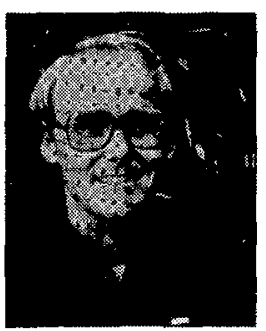

Bjorn Petersen was born in Denmark, in 1948. He received the M.Sc. degree in electrical engineering from the Technical University of Denmark, Lyngby, Denmark, in 1976, and is currently working towards the $\mathrm{Ph}$.D. degree at the Institute of Circuit Theory and Telecommunication, and the Department of Mathematics, Technical University of Denmark.

Until February 1977, he was an Associate Professor at the Department of Mathematics, University of Denmark, Lyngby. He has carried out research in the fields of circuit theory, graph theory, matroid theory, and computational complexity. His fields of interest are linear active network theory and matroid algorithms.

\title{
Realization Theory of Discrete-Time Nonlinear Systems: Part I - The Bounded Case
}

\author{
EDUARDO D. SONTAG
}

\begin{abstract}
A state-space realization theory is presented for a wide class of discrete time input/output behavions. Although in many ways restricted, this class does include as particular cases those treated in the literature (linear, multilinear, internally bilinear, homogeneous), as well as certain nonanalytic nonlinearities. The theory is conceptually simple, and matrixtheoretic algorithms are straightforward. Finite-realizability of these behaviors by state-affine systems is shown to be equivalent both to the existence of high-order input/output equations and to realizability by more general types of systems.
\end{abstract}

\section{INTRODUCTION}

$\mathrm{T}$ HIS WORK deals with some aspects of realization theory of deterministic nonlinear discrete-time systems. The realization theory of linear systems is by now a

Manuscript received September 11, 1977; revised July 6, 1978. This research was supported in part by U. S. Army Research Grant DAH C04-74-G-0153 through the Center for Mathematical System Theory, Gainesville, FL 32611 .

The author is with the Department of Mathematics, Rutgers University, New Brunswick, NJ 08903. successful part of system theory, which has resulted in a deep understanding of behavior and has permitted the application of state-space methods of analysis and synthesis. It may be reasonable to expect, then, that a corresponding theory will eventually derive analogous benefits for nonlinear systems.

For the most part, this paper presents a "linearized" realization theory via systems which are linear in state variables but arbitrarily nonlinear in inputs, state-affine systems. While such systems are highly restrictive vis à vis general nonlinear models, they do include those for which a detailed realization theory has been developed, in particular, linear, internally bilinear, and multilinear systems. The importance of S-A representations in the analysis of certain nuclear reactors, heat-transfer processes, and population models, among others, has been made explicit by various authors (see, for instance, [34]); other applications being currently explored are in the areas of image processing and in stochastic filtering. Moreover, in some 